\title{
Association between FGF19, FGF21 and lipocalin-2, and diabetes progression in PCOS
}

\author{
Feifei Cheng ${ }^{1}$, Noel Yat Hey Ng${ }^{1}$, Claudia Ha Ting Tam ${ }^{1,2}$, Yuying Zhang ${ }^{1,2}$, Cadmon King Poo Lim ${ }^{1,3}$, Guozhi Jiang ${ }^{1,2}$, \\ Alex Chi Wai Ng', Tiffany Tse Ling Yau', Lai Ping Cheung ${ }^{4}$, Aimin Xu ${ }^{5,6,7}$, Juliana C N Chan ${ }^{1,2,3,8}$ and Ronald C W Ma ${ }^{1,2,3,8}$ \\ 'Department of Medicine and Therapeutics, The Chinese University of Hong Kong, Hong Kong, Hong Kong \\ ${ }^{2}$ Hong Kong Institute of Diabetes and Obesity, The Chinese University of Hong Kong, Hong Kong, Hong Kong \\ ${ }^{3}$ Li Ka Shing Institute of Health Sciences, The Chinese University of Hong Kong, Hong Kong, Hong Kong \\ ${ }^{4}$ Department of Obstetrics and Gynaecology, The Chinese University of Hong Kong, Hong Kong, Hong Kong \\ ${ }^{5}$ Department of Medicine, Li Ka Shing (LKS) Faculty of Medicine, University of Hong Kong, Hong Kong, Hong Kong \\ ${ }^{6}$ State Key Laboratory of Pharmaceutical Biotechnology, University of Hong Kong, Hong Kong, Hong Kong \\ ${ }^{7}$ Department of Pharmacy and Pharmacology, LKS Faculty of Medicine, University of Hong Kong, Hong Kong, Hong Kong \\ ${ }^{8}$ Chinese University of Hong Kong-Shanghai Jiao Tong University Joint Research Centre in Diabetes Genomics and Precision Medicine, Hong Kong, Hong Kong
}

Correspondence should be addressed to R C W Ma: rcwma@cuhk.edu.hk

\begin{abstract}
Women with polycystic ovary syndrome (PCOS) have an increased risk of developing type 2 diabetes. FGF19, FGF21 and lipocalin-2 have emerged as important markers of metabolic risk. This study aims to compare the levels of FGF19, FGF21 and lipocalin-2 between subjects with or without PCOS, and to investigate the relationship between proteins and diabetes progression. In this nested case-control cohort study, 128 Chinese PCOS women and 128 controls were recruited and followed-up. All subjects underwent the oral glucose tolerance test for the evaluation of glycaemic status. Baseline serum protein levels were measured using ELISA. Compared with controls, PCOS subjects had higher levels of FGF19 $(P<0.001)$ and FGF21 $(P=0.022)$, but had lower lipocalin-2 $(P<0.001)$. In total, $20.8 \%$ of PCOS and $9.2 \%$ of controls developed diabetes over a mean duration of $10.4 \pm 1.2$ and $11.3 \pm 0.5$ years, respectively. Logistic regression analyses suggested FGF19 was positively associated with diabetes progression in controls, after adjusting for age, follow-up duration, waist and fasting glucose $(P=0.026$, odds ratio (OR) $(95 \% \mathrm{Cl}): 7.4(1.3-43.6)$ ), and the positive relationship between FGF21 and diabetes progression in controls was attenuated by adjusting for age and follow-up duration $(P=0.183)$. Lipocalin-2 was positively correlated with diabetes progression in PCOS group $(P=0.026$, OR $(95 \% \mathrm{Cl}))$ : $2.5(1.1-5.6)) ;$ however, this became attenuated after adjusting for waist and fasting glucose $(P=0.081)$. In conclusion, there is differential expression of FGF19, FGF21, and lipocalin-2 in PCOS. The serum level of FGF19, and FGF21 is associated with diabetes progression in women without PCOS, while lipocalin- 2 was related to diabetes progression in PCOS women.
\end{abstract}

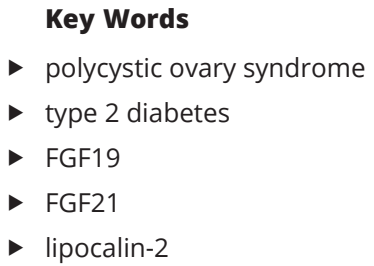

Endocrine Connections (2021) 10, 1243-1252

\section{Introduction}

Polycystic ovary syndrome (PCOS) is a common endocrine disorder among women of reproductive age, affecting about $6-12 \%$ of the overall female population (1). A large community-based study in China reported a prevalence rate of $5.6 \%$ in women aged 19-45 years old (2).
Women with PCOS have several clinical characteristics including hyperandrogenism, obesity, insulin resistance (IR), pancreatic $\beta$-cell dysfunction and abnormal glucose metabolism, which may explain the increased risk of type 2 diabetes (T2DM) in PCOS patients $(3,4)$.
This work is licensed under a Creative Commons Attribution-NonCommercial-NoDerivatives 4.0 enternationab ticense.ifica.com at 04/26/2023 02:07:18PM 
To date, some studies have reported the high progression rate of T2DM in PCOS patients. Gambineri et al. followed a total of 255 PCOS women for a mean duration of 10 years and found the incidence rate of T2DM was 1.05 per 100 person-years (5). The age-standardized prevalence of diabetes at the end of follow-up was 39.3\%, which was significantly higher than that of women of a similar age in the general population in Italy (5.8\%) (5). In another study from Denmark, over a median 11.1 years' follow-up, the incidence rate of T2DM was 8.0 per 1000 person-years in PCOS, compared to 2 per 1000 personyears in controls $(P<0.001)(6)$. The authors found that BMI, glucose, IR, lipids and sex hormone-binding globulin (SHBG) were associated with the development of T2DM in PCOS patients $(5,6)$. In a long-term follow-up study from Hong Kong, the incidence rate of diabetes in PCOS was 22.12 per 1000 person-years, which was more than doubled that of women without PCOS (10.09 per 1000 person-years) (7).

In addition to established clinical risk factors, other biomarkers have emerged as important predictors of diabetes risk $(8,9)$. Two members from the fibroblast growth factor (FGF) family, FGF19 and FGF21, have been shown to be associated with obesity and diabetes $(10,11$, 12). Moreover, serum FGF21 can predict the development of T2DM in the normal population, as well as being associated with incident coronary heart disease (CHD) and kidney disease in diabetes $(13,14)$. Besides, lipocalin-2, a well-recognized biomarker of inflammation, infection, as well as kidney damage, may accelerate the development of diabetes by promoting IR and inflammation (15). However, although FGF19, FGF21, and lipocalin-2 have been linked with diabetes risk, whether they are associated with progression of diabetes in patients with PCOS is not known.

The aims of our study include (1) to compare the levels of FGF19, FGF21, and lipocalin-2 between PCOS and healthy controls, and (2) to investigate for any possible association between baseline FGF19, FGF21, and lipocalin-2 with the progression of diabetes in PCOS subjects and controls.

\section{Materials and methods}

\section{Subjects}

In this nested case-control cohort study, 128 Chinese women with PCOS were included from the PCOS registry at the Prince of Wales Hospital (PWH), recruited between July 2003 and April 2007. The diagnosis of PCOS was based on the 2003 Rotterdam consensus (at least two of the following criteria: oligo- or anovulation, biochemical and/or clinical androgen excess, and polycystic ovary appearance by ultrasound) (16). We also included 128 controls from a population-based healthy women cohort recruited between 2003 and 2006 (17). All control subjects had regular menstrual cycles and had no hirsutism or ultrasound features of polycystic ovaries. Exclusion criteria for both groups included the use of oral contraceptives or corticosteroids within the past 3 months, and subjects who were previously diagnosed with hypothyroidism, prolactinoma, non-classical adrenal hyperplasia and Cushing's syndrome. Between January 2016 and December 2017, all PCOS controls were recalled to visit the Diabetes Mellitus \& Endocrine Research Centre at PWH for detailed clinical and metabolic evaluation as described previously (7). Control subjects were invited to participate in a similar follow-up evaluation approximately 10 years after the baseline assessment. Nine control subjects failed to complete the follow-up assessment because of refusal $(n=8)$, or failure to be contacted $(n=1)$. The study was approved by the Joint Chinese University of Hong KongNew Territories East Cluster Clinical Research Ethics Committee (CREC ref. no. 2015.040), and informed written consent was obtained from all subjects.

\section{Clinical, anthropometrical and biochemical parameters}

All subjects at baseline and follow-up were assessed by well-trained research nurses. A standard questionnaire was used to document personal records including medical and drug history. Blood pressure and anthropometric measurements, including height $(\mathrm{cm})$, weight $(\mathrm{kg})$, waist circumference $(\mathrm{cm})$ and hip circumference $(\mathrm{cm})$, were obtained from both groups at baseline and follow-up. BMI and waist-to-hip ratio (WHR) were calculated accordingly.

Overnight fasting blood was obtained in all women to measure fasting plasma glucose (FPG), total cholesterol (TC), triglycerides (TG), low-density lipoprotein cholesterol (LDL-C), high-density lipoprotein cholesterol (HDL-C) and estimated glomerular filtration rate (eGFR) using the Chronic Kidney Disease Epidemiology Collaboration $(\mathrm{CKD}-\mathrm{EPI})$ formula: $\mathrm{eGFR}=141 \times \min (\mathrm{Scr} / \kappa, 1) \propto \times \max$ $(\mathrm{Scr} / \varkappa, 1)-1.209 \times 0.993$ age $\times 1.018$ (if female) $\times 1.159$ (if black) (18). Fasting insulin, female sex hormones, and antiMüllerian hormone (AMH) were also measured. A standard $75 \mathrm{~g}$ oral glucose tolerance test (OGTT) was conducted in all subjects, unless they had already been diagnosed with T2DM earlier. Insulin resistance was estimated using

This work is licensed under a Creative Commons Attribution-NonCommercial-NoDerivatives 4.0 enternationad License.ifica.com at 04/26/2023 02:07:18PM 
the homeostasis model assessment for insulin resistance (HOMA-IR) by dividing the product of fasting insulin and glucose by 22.5 . HOMA- $\beta$ was calculated by the following formula: $(20 \times$ fasting insulin $(\mathrm{mIU} / \mathrm{mL}))$, (FPG $(\mathrm{mmol} / \mathrm{L})-3.5)$. The diagnosis of T2DM and dysglycaemia was according to the 2009 ADA diagnostic criteria (19): (1) impaired fasting glucose (IFG), fasting plasma glucose 5.6-6.9 mmol; (2) impaired glucose tolerance (IGT), 2-h plasma glucose $7.8-11.0 \mathrm{mmol}$; (3) DM, FPG $\geq 7.0 \mathrm{mmol}$, or symptoms of hyperglycaemia and a random plasma glucose $\geq 11.1 \mathrm{mmol}$, or 2 -h plasma glucose $\geq 11.1 \mathrm{mmol}$ during OGTT, or self-reported history of DM. The diabetes converters were defined as the occurrence of diabetes in normal (or IFG, IGT) subjects. Hypertension was diagnosed for subjects with systolic blood pressure (SBP) $\geq 140 \mathrm{~mm}$ $\mathrm{Hg}$, or diastolic blood pressure (DBP) $\geq 90 \mathrm{~mm} \mathrm{Hg}$, or if on anti-hypertensive medications. Subjects with $\mathrm{TC} \geq 5.2$ mmol, TG $\geq 1.7 \mathrm{mmol}$, LDL-C $\geq 3.4 \mathrm{mmol}$, HDL-C $\leq 1.3$ $\mathrm{mmol}$, or those on lipid-lowering therapies were diagnosed as having dyslipidaemia. Signs of hyperandrogenism were noted in the physical examination, and the modified Ferriman-Gallwey score was used to diagnose clinical hirsutism. The cut-off point was defined as $\geq 3$ based on the guideline $(20,21)$.

\section{Laboratory analyses}

Plasma glucose, TC, TG, LDL-C, and HDL-C concentration were measured by DP Modular Analytics (Roche Diagnostics Corp.). Serum levels of luteinizing hormone (LH), follicle-stimulating hormone (FSH), total oestradiol, and total testosterone levels were measured by electrochemiluminescent immunoassays on the Roche E170 system (RocheDiagnostics Corp.).SHBG was measured using Immulite 1000 Analyser (Siemens Healthcare Diagnostics Inc.). Free androgen index was calculated by $100 \times$ (total testosterone $(\mathrm{nmol}) /$, SHBG $(\mathrm{nmol}))$. AMH was measured using Elecsys ${ }^{\circledast}$ AMH electro-chemiluminescent immunoassay Roche Cobas E411 analyser (Roche Diagnostics Corp.). Insulin levels in the PCOS group were tested using an automated chemiluminescent immunoassay analyser (Siemens Healthcare Diagnostics Inc.), while ELISA kits (Dako Denmark A/S) were used to measure the insulin levels of the control group). The levels of FGF19, FGF21, and lipocalin-2 were measured by the corresponding ELISA kits (Antibody and Immunoassay Services, Hong Kong). These measurements were performed using standard reagent kits supplied by the manufacturers. The analytical performance of these assays was within the specifications of the analysers.

\section{Statistical analysis}

Data are expressed as mean \pm S.D., or median (Q1-Q3), according to the distribution of each variable. Covariates were categorized or natural logarithm transformed if needed. Comparison between groups was performed using Student's $t$-test or Mann-Whitney $U$-test, as appropriate. When comparing the difference of baseline characteristics between PCOS women and healthy controls, all analyses were adjusted for the baseline age in either general linear regression (for continuous data) or logistic regression (for categorical data). Chi-square $\left(\chi^{2}\right)$ or Fisher's exact tests were used as appropriate for categorical variables to examine the difference of frequencies between groups. The paired $t$-test or Wilcoxon signed ranks test was used to compare continuous variables between the baseline and follow-up visit.

To test the joint effects of biomarkers on PCOS risk, we defined the threshold using the median level of FGF19, FGF21 and lipocalin-2 in the control group, and assigned a score of 1 to each of (i) increased FGF19, (ii) increased FGF21, and (iii) decreased lipocalin-2, to generate a score with a maximum of 3 , representing the total number of abnormal biomarkers in the combined analysis. We also applied regression analyses (linear regression for continuous variables or logistic regression for categorical variables) to test the relationship between the total number of abnormal biomarkers and clinical variables in combined datasets, after adjustment for age and BMI at baseline.

General linear models were used to compare the differences in the levels of FGF19, FGF21 and lipocalin-2 between diabetes converters and non-converters in PCOS women and controls, respectively. Multivariate logistic regression analysis was used to explore the independent effects of the biomarkers on diabetes progression among subjects with or without PCOS. Due to our limited sample size, we adjusted for age and waist circumference in our main analysis for the association between the biomarkers and PCOS (model 2), and included adjustment for age and follow-up duration for incident diabetes as the outcome (model 2), but we have also undertaken exploratory analyses adjusting for additional covariates. The Q-Test of heterogeneity was used to investigate if the effect sizes of the different biomarkers derived from PCOS and control groups are different from each other. $P \leq 0.05$ (two-tailed) was considered to be statistically significant. All statistical analyses were performed with the Statistical Package for the Social Sciences for Windows, version 24.0 (SPSS Inc.).

This work is licensed under a Creative Commons Attribution-NonCommercial-NoDerivatives 4.0 Internationab bicense ifica com at $04 / 26 / 2023 \quad 02: 07: 18 \mathrm{PM}$ 


\section{Results}

\section{Comparison between women with or without PCOS at baseline}

As the recruited PCOS subjects were significantly younger than the healthy controls ( $30.0 \pm 6.4$ vs $42.8 \pm 7.2, P<0.001$ ), all comparisons of baseline characteristics between PCOS and controls were adjusted for age. After adjustment, PCOS women at baseline were more obese, with higher BMI, waist, and WHR, as well as with higher TG, FPG, 2-h glucose, HOMA-IR, AMH and eGFR, but lower LDL-C, compared to controls. There was no significant difference in blood pressure, TC, HDL-C, insulin levels, and HOMA- $\beta$ between PCOS and controls (Table 1).

\section{Comparison between baseline and follow-up}

After $10.4 \pm 1.2$ years' follow-up, PCOS women became more overweight with increased BMI and WHR. There was a significant tendency towards an increase in blood pressure, while TC TG and LDL-C were also increased along with FPG 2-h glucose and FSH. However, insulin and HOMA- $\beta$ were decreased at the follow-up. The same tendency of increased BMI WHR blood pressure lipids FPG $2 \mathrm{~h}$ glucose and decreased HOMA- $\beta$ were also observed in the control group (Supplementary Table 2, see section on supplementary materials given at the end of this article).

In total, $9.2 \%(11,119)$ healthy subjects developed diabetes during follow-up, whilst others remained normal. Meanwhile, among 128 PCOS subjects, 6.3\% (8128) were diagnosed with diabetes at baseline. During the follow-up period, $79.2 \%(95,120)$ remained free of diabetes, while $20.8 \%(25,120)$ progressed to diabetes. Compared with the corresponding non-converters, both converters in the control and PCOS groups were more overweight at baseline, with higher baseline BMI and waist, as well as higher FPG.

\section{Novel biomarkers in PCOS vs controls}

Adjustment of age and waist were included in the subsequent analyses, given the significant differences in age and obesity between PCOS and control group. PCOS subjects showed significantly higher levels of FGF19 (192 (105-308) vs $124(61-191), P<0.001)$ and FGF21 (154 (74.5-266) vs $103.7(50-173), P=0.022)$, but lower levels of lipocalin-2 (56.1 (35.7-75.9) vs 94.0 (63.1-162.4), $P<0.001$ ) (Table 1).

In logistic regression analyses of the relationship between the levels of the three serum proteins and
PCOS status, FGF19 and FGF21 were both positively associated with PCOS status (odds ratio (OR) (95\% CI): 2.0 (1.5-2.7) and $1.4(1.1-1.8)$, respectively, $P<0.001$ and $P=0.010$ ), while lipocalin- 2 showed negative association (OR (95\% CI): $0.2(0.1-0.3), P<0.001)$. After adjusting for variables with a significant difference at baseline (including age, waist, FPG and TG, AMH, hypertension, and dyslipidaemia at baseline), the difference of FGF19 and lipocalin-2 between PCOS and control remained significant. However, the association between FGF21 and PCOS status became attenuated after further adjusting for FPG and lipids (Table 2).

We also explored the joint effects of FGF19, FGF21 and lipocalin-2 on PCOS risk in the combined cohort. The median level of each protein in the control population was used as the cut-off point, and we assigned either FGF19 > $123.7 \mathrm{pg} / \mathrm{mL}$, FGF21 > $103.7 \mathrm{pg} / \mathrm{mL}$ or lipocalin-2 $<94.0 \mathrm{ng} / \mathrm{mL}$ as one abnormal biomarker and calculated the total number of abnormal biomarkers for each subject, which was linearly associated with a diagnosis of PCOS (OR (95\% CI): 2.2 (1.6-3.0), $P<0.001)$. The association was still significant (OR (95\% CI): 3.7 (2.1-6.5), $P<0.001$ ) even after adjusting for age and waist at baseline. When subjects were stratified according to the number of abnormal biomarkers, namely $\leq 1,2$ and 3, with similar sample sizes in each group, those with the maximum number of abnormal biomarkers, which accounted for $28.5 \%$ of the study population, had 5.2-fold higher risk for PCOS compared to those with abnormal biomarkers $\leq 1(P<0.001$, Table 3$)$. Increased number of abnormal biomarkers was associated with SBP $(P=0.034)$, hypertension $(P=0.035)$, hyperandrogenism $(P=0.017)$ and AMH $(P=0.003$, Supplementary Table 3$)$ in exploratory analyses.

\section{FGF19, FGF21 and lipocalin-2 in diabetes converters vs non-converters}

Compared with non-converters in the control group, DM converters had significantly higher baseline FGF19 (189 (77-374) vs 122 (57-187), $P=0.036)$ and FGF21 (232 (142-315) vs 90 (48-159), $P=0.002$ ). After adjusting for age, waist and HOMO-IR, the difference in FGF21 was still significant $(P=0.010)$, but the difference in FGF19 was rendered non-significant $(P=0.063)$. In the PCOS group, DM converters had significantly higher levels of lipocalin-2 at baseline (70.6 (42.3-99.2) vs 51.9 (33.5-65.6), $P=0.022$ ); however, after adjusting for age, waist and HOMO-IR, this became non-significant $(P=0.056)$.

This work is licensed under a Creative Commons Attribution-NonCommercial-NoDerivatives 4.0 Internationad bicense.ifica.com at 04/26/2023 02:07:18PM 
Table 1 The comparison of clinical characteristics between PCOS and control group at baseline. Data are expressed as mean \pm S.D., median (Q1-Q3), or proportion in percentage. All comparison was adjusted for the differences of age by using either general linear model for continuous data or logistic regression model for categorical data. Ln transformation were used in triglyceride, insulin, HOMA-IR, HOMA- $\beta$, AMH, FGF19, FGF21 and lipocalin-2.

\begin{tabular}{|c|}
\hline Baseline variables \\
\hline Age (years) \\
\hline BMI $\left(\mathrm{kg} / \mathrm{m}^{2}\right)$ \\
\hline waist $(\mathrm{cm})$ \\
\hline Waist-to-hip ratio \\
\hline $\mathrm{SBP}(\mathrm{mmHg})$ \\
\hline $\mathrm{DBP}(\mathrm{mmHg})$ \\
\hline Total cholesterol (mmol/L) \\
\hline Triglyceride (mmol/L) \\
\hline $\mathrm{HDL}-\mathrm{C}(\mathrm{mmol} / \mathrm{L})$ \\
\hline LDL-C (mmol/L) \\
\hline Glu-fast (mmol/L) \\
\hline Glu-2 h (mmol/L) \\
\hline Insulin $(\mu \mathrm{U} / \mathrm{mL})$ \\
\hline HOMA-IR \\
\hline HOMA- $\beta$ \\
\hline eGFR $\left(\mathrm{mL} / \mathrm{min} / 1.73 \mathrm{~m}^{2}\right)$ \\
\hline AMH (pmol/L) \\
\hline LH (IU/L) \\
\hline FSH (IU/L) \\
\hline LH/FSH \\
\hline Testosterone (nmol/L) \\
\hline Hirtusim (\%) \\
\hline Oestrogen (pmol/L) \\
\hline Use of metformin (\%) \\
\hline Use of anti-hypertension drugs (\%) \\
\hline Use of lipid-lowering drug \\
\hline IGR (\%) \\
\hline DM (\%) \\
\hline $\mathrm{HT}(\%)$ \\
\hline Dyslipidaemia (\%) \\
\hline FGF19 (pg/mL) \\
\hline FGF21 (pg/mL) \\
\hline Lipocalin-2 (ng/mL) \\
\hline
\end{tabular}

\begin{tabular}{|c|}
\hline Control $(n=128)$ \\
\hline $42.8 \pm 7.2$ \\
\hline $23.1 \pm 3.6$ \\
\hline $75.3 \pm 8.3$ \\
\hline $0.8 \pm 0.1$ \\
\hline $111 \pm 15$ \\
\hline $71 \pm 10$ \\
\hline $5.1 \pm 1.1$ \\
\hline $0.8(0.6-1.2)$ \\
\hline $1.7 \pm 0.5$ \\
\hline $2.9 \pm 1.0$ \\
\hline $4.8 \pm 0.5$ \\
\hline $6.3 \pm 1.8$ \\
\hline $7.4(4.9-10.8)$ \\
\hline $1.6(1.0-2.4)$ \\
\hline $121(76-203)$ \\
\hline $95 \pm 16.3$ \\
\hline $1.8(0.1-7.6)$ \\
\hline- \\
\hline- \\
\hline- \\
\hline- \\
\hline- \\
\hline $0 / 128$ \\
\hline $0 / 128$ \\
\hline $0 / 128$ \\
\hline $19 / 128(14.8)$ \\
\hline $0 / 128$ \\
\hline $11 / 128$ (8.6) \\
\hline $86 / 128(67.2)$ \\
\hline $124(61-191)$ \\
\hline $104(50-173)$ \\
\hline $94.0(63.1-162.4)$ \\
\hline
\end{tabular}

\begin{tabular}{|c|c|}
\hline $\operatorname{PCOS}(n=128)$ & $P$ value \\
\hline $30.0 \pm 6.4$ & - \\
\hline $25.9 \pm 5.8$ & 0.001 \\
\hline $82.5 \pm 13.0$ & $<0.001$ \\
\hline $0.8 \pm 0.1$ & $<0.001$ \\
\hline $111.2 \pm 15$ & 0.140 \\
\hline $71 \pm 10$ & 0.253 \\
\hline $4.8 \pm 1.1$ & 0.196 \\
\hline $0.9(0.7-1.5)$ & 0.005 \\
\hline $1.6 \pm 0.6$ & 0.767 \\
\hline $2.6 \pm 0.9$ & 0.016 \\
\hline $5.1 \pm 1.1$ & 0.002 \\
\hline $7.3 \pm 2.8$ & 0.011 \\
\hline $10.5(6.2-22.6)$ & 0.054 \\
\hline $2.3(1.3-5.5)$ & 0.019 \\
\hline 153 (93-296) & 0.749 \\
\hline $111.9 \pm 13.2$ & 0.046 \\
\hline 27.5 (19.4-45.2) & $<0.001$ \\
\hline \multicolumn{2}{|l|}{$8.6 \pm 6.5$} \\
\hline \multicolumn{2}{|l|}{$5.6 \pm 2.0$} \\
\hline \multicolumn{2}{|l|}{$1.6 \pm 1.1$} \\
\hline \multicolumn{2}{|l|}{$1.6(1.2-2.3)$} \\
\hline \multicolumn{2}{|l|}{ 16/126 (12.7) } \\
\hline \multicolumn{2}{|l|}{$141(112-222)$} \\
\hline \multicolumn{2}{|l|}{$12 / 128(9.4)$} \\
\hline \multicolumn{2}{|l|}{$5 / 128(3.9)$} \\
\hline \multicolumn{2}{|l|}{$0 / 128$} \\
\hline $32 / 128(25.0)$ & 0.057 \\
\hline $8 / 128(6.3)$ & \\
\hline $23 / 128(18.0)$ & 0.013 \\
\hline $64 / 128(50.0)$ & 0.285 \\
\hline $192(105-308)$ & $<0.001$ \\
\hline $154(75-266)$ & 0.022 \\
\hline $56.1(35.7-75.9)$ & $<0.001$ \\
\hline
\end{tabular}

$\mathrm{AMH}$, anti-Mullerian hormone; DBP, diastolic blood pressure; eGFR, estimated glomerular filtration rate; FSH, follicle-stimulating hormone; Glu-2h, 2-h glucose; Glu-fast, fasting glucose; HDL-C, high-density lipoprotein cholesterol; HOMA-IR, homeostasis model assessment of insulin resistance; HOMA- $\beta$, homeostasis model assessment of beta-cell function; HT, hypertension; IGR, impaired glucose regulation; LDL-C, low-density lipoprotein cholesterol; LH, luteinizing hormone; SBP, systolic blood pressure.

Binary logistic regression found that the level of serum FGF19 was positively associated with the progression of diabetes in the control group $(P=0.029$, OR $(95 \% \mathrm{CI})$ : 2.4 (1.1-5.3)). When further adjusting for age, follow-up duration, baseline waist and FPG, the association was still significant $(P=0.026$, OR (95\% CI): $7.4(1.3-43.6))$. Serum FGF21 concentration also suggested positive relationship of diabetes progression in women without

Table 2 Logistic regression models for the relationship between FGF19, FGF21 and lipocalin-2, and PCOS status.

\begin{tabular}{|c|c|c|c|c|c|c|c|c|}
\hline \multirow[b]{2}{*}{ Biomarkers } & \multicolumn{2}{|c|}{ Model 1} & \multicolumn{2}{|c|}{ Model 2} & \multicolumn{2}{|c|}{ Model 3} & \multicolumn{2}{|c|}{ Model 4} \\
\hline & OR $(95 \% \mathrm{Cl})$ & $P$ & OR $(95 \% \mathrm{Cl})$ & $P$ & OR $(95 \% \mathrm{Cl})$ & $P$ & OR $(95 \% \mathrm{Cl})$ & $P$ \\
\hline Ln (FGF19) & $2.0(1.5-2.7)$ & $<0.001$ & $2.5(1.5-4.0)$ & $<0.001$ & $3.1(1.5-6.6)$ & 0.003 & $3.4(1.5-7.3)$ & 0.002 \\
\hline Ln (FGF21) & $1.4(1.1-1.8)$ & 0.010 & $1.7(1.1-2.5)$ & 0.018 & $1.5(0.9-2.6)$ & 0.108 & $1.6(0.9-2.7)$ & 0.096 \\
\hline Ln (lipocalin-2) & $0.2(0.1-0.3)$ & $<0.001$ & $0.2(0.1-0.3)$ & $<0.001$ & $0.2(0.1-0.5)$ & $<0.001$ & $0.2(0.1-0.5)$ & 0.001 \\
\hline
\end{tabular}

Ln, natural logarithm; Model 1, no adjustment; Model 2, Model 1 + adjusted for age and waist at baseline; Model 3, Model 2 + adjusted for fasting glucose, LN (triglyceride), and AMH at baseline; Model 4, Model $3+$ adjusted for hypertension and dyslipidaemia at baseline.

https://ec.bioscientifica.com https://doi.org/10.1530/EC-21-0082 (c) 2021 The authors Published by Bioscientifica Ltd

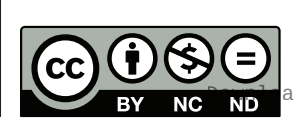

This work is licensed under a Creative Commons Attribution-NonCommercial-NoDerivatives 4.0 Internationab ficense.ifica . com at $04 / 26 / 2023 \quad 02: 07: 18 \mathrm{PM}$ 
Table 3 Logistic regression for the relationship between joint effects of FGF19, FGF21 and lipocalin-2, and PCOS status.

\begin{tabular}{l}
\hline \\
\hline No. of abnormal \\
biomarkers \\
No. of abnormal \\
biomarkers $(\leq 1)$ \\
No. of abnormal \\
biomarkers (2) \\
No. of abnormal \\
biomarkers (3)
\end{tabular}

$\frac{\text { Model } 1}{\frac{\mathrm{OR}(95 \% \mathrm{Cl})}{2.2(1.6-3.0)} \frac{P}{<0.001}}$

Reference

$3.0(1.6-5.6) \quad 0.001$

$5.2(2.6-10.4)<0.001$

$\frac{\text { Model } 2}{\frac{\text { OR }(95 \% \mathrm{Cl})}{3.7(2.1-6.5)} \frac{P}{<0.001}}$

Reference

$5.3(1.9-14.7)$

$14.1(4.3-46.8)$

$<0.001$

\section{4}

$\frac{\text { Model } 3}{\frac{\text { OR }(95 \% \mathrm{Cl})}{4.4(2.0-9.9)}} \frac{P}{<0.001}$

$\frac{\text { Model 4 }}{\frac{\mathrm{OR}(95 \% \mathrm{Cl})}{4.4(1.9-9.9)}} \frac{P}{<0.001}$

Reference

$4.3(1.1-17.3) \quad 0.041$

$4.4(1.0-18.8)$

0.043

$17.0(3.3-88.5)$

0.001

$16.5(3.1-87.0)$

0.001

Model 1, no adjustment; Model 2, Model 1 + adjusted for age and waist at baseline; Model 3, Model 2 + adjusted for fasting glucose, LN (triglyceride), and AMH at baseline; Model 4, Model $3+$ adjusted for hypertension and dyslipidaemia at baseline. Abnormal biomarkers were defined as FGF19>123.7 pg/mL, FGF21 $>103.7 \mathrm{pg} / \mathrm{mL}$, or lipocalin-2 $<94.0 \mathrm{ng} / \mathrm{mL}$ using the median level of control group. The number of abnormal biomarkers was counted for each subject and categorized by $\leq 1,2$ and 3 . Group with abnormal biomarkers $\leq 1$ was used as a reference in logistic regression models.

PCOS $(P=0.004$, OR (95\% CI): $3.9(1.5-10.1))$; however, the association became attenuated after adjusting for baseline age and follow-up duration $(P=0.183$, OR $(95 \% \mathrm{CI}): 3.4$ (0.6-19.9)). No significant association between FGF19 (or FGF21) and diabetes progression was observed in the PCOS group (Table 4).

The relationship between serum level of lipocalin-2 and progression of diabetes was positive in the PCOS group $(P=0.026$, OR (95\% CI): 2.5 (1.1-5.6)), but was negative in the control group, though not statistically significant $(P=0.396$, OR (95\% CI): 0.6 (0.2-1.8)). After adjusting for age and follow-up duration, the relationship remained significant ( $P=0.025$ and 0.005 , respectively). When further adjusting for baseline waist and FPG, the relationship in the control group still existed $(P=0.042$, OR (95\% CI): $0.1(0-0.9))$, but was no longer significant in the PCOS group $(P=0.081)$. The Q-test of heterogeneity showed the OR of serum lipocalin-2 was significantly different between women with and without PCOS
$(P=0.009)$, even after adjusting for age, follow-up duration, baseline waist and FPG (Table 4).

\section{Sensitivity analysis}

Logistic regression models were also constructed to explore the relationship between FGF19, FGF21 and lipocalin-2, and progression to pre-diabetes or diabetes in women with or without PCOS. A total of $26.6 \%$ subjects in the control group and $37.5 \%$ subjects in PCOS developed diabetes from normal, pre-diabetes, or pre-diabetes from normal status. FGF21 was significantly associated with progression to pre-diabetes or diabetes in the control group $(P=0.038, \mathrm{OR}$ (95\% CI): 1.6 (1-2.6)). When further adjusting for baseline waist circumference, the association became attenuated $(P=0.061)$. In the PCOS group, lipocalin-2 was positively associated with progression to dysglycaemia, which persisted even after adjusting for age, waist and FPG $(P=0.047$, OR (95\% CI): 2.6 (1-6.7)) (Supplementary Table 4).

Table 4 Logistic regression models for the risk of progression to diabetes in women with or without PCOS.

\begin{tabular}{|c|c|c|c|c|c|c|}
\hline \multirow[b]{2}{*}{ Model } & \multirow[b]{2}{*}{ Biomarkers } & \multicolumn{2}{|c|}{ Pcos } & \multicolumn{2}{|c|}{ Control } & \multirow{2}{*}{$\frac{\text { Q-test }}{P}$} \\
\hline & & OR $(95 \% \mathrm{Cl})$ & $P$ & OR $(95 \% \mathrm{Cl})$ & $P$ & \\
\hline \multirow[t]{3}{*}{ Model 1} & $\operatorname{Ln}(\mathrm{FGF19)}$ & $0.8(0.5-1.3)$ & 0.299 & $2.4(1.1-5.3)$ & 0.029 & 0.016 \\
\hline & $\operatorname{Ln}(\mathrm{FGF} 21)$ & $1.3(0.8-2.2)$ & 0.334 & $3.9(1.5-10.1)$ & 0.004 & 0.040 \\
\hline & Ln (lipocalin-2) & $2.5(1.1-5.6)$ & 0.026 & $0.6(0.2-1.8)$ & 0.396 & 0.042 \\
\hline \multirow[t]{3}{*}{ Model 2} & Ln (FGF19) & $0.8(0.5-1.3)$ & 0.388 & $6.0(1.4-26.5)$ & 0.018 & 0.012 \\
\hline & $\operatorname{Ln}(\mathrm{FGF} 21)$ & $1.1(0.6-2.0)$ & 0.651 & $3.4(0.6-19.9)$ & 0.183 & 0.258 \\
\hline & Ln (lipocalin-2) & $2.9(1.1-7.3)$ & 0.025 & $0.2(0-0.9)$ & 0.043 & 0.005 \\
\hline \multirow[t]{3}{*}{ Model 3} & Ln (FGF19) & $0.9(0.5-1.6)$ & 0.730 & $6.3(1.4-29.1)$ & 0.019 & 0.020 \\
\hline & $\operatorname{Ln}(F G F 21)$ & $1.3(0.7-2.4)$ & 0.376 & $3.3(0.6-19.7)$ & 0.189 & 0.333 \\
\hline & Ln (lipocalin-2) & $2.4(0.9-6.4)$ & 0.081 & $0.1(0-0.9)$ & 0.034 & 0.008 \\
\hline \multirow[t]{3}{*}{ Model 4} & Ln (FGF19) & $1.0(0.5-2.1)$ & 0.892 & $7.4(1.3-43.6)$ & 0.026 & 0.043 \\
\hline & $\operatorname{Ln}(\mathrm{FGF} 21)$ & $1.4(0.7-2.6)$ & 0.330 & $2.6(0.5-13.8)$ & 0.267 & 0.489 \\
\hline & Ln (lipocalin-2) & $2.6(0.9-7.7)$ & 0.081 & $0.1(0-0.9)$ & 0.042 & 0.009 \\
\hline
\end{tabular}

Ln, natural logarithm; Model 1, no adjustment; Model 2, Model 1 + adjusted for age and follow-up duration; Model 3, Model 2 + adjusted for waist at baseline; Model 4, Model 3 + adjusted for fasting glucose at baseline. Q-Test of heterogeneity to investigate if effect sizes of biomarkers derived from PCOS and control are significantly different from each other.

https://ec.bioscientifica.com https://doi.org/10.1530/EC-21-0082 (c) 2021 The authors Published by Bioscientifica Ltd

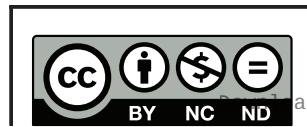

This work is licensed under a Creative Commons Attribution-NonCommercial-NoDerivatives 4.0 International License ifica com at $04 / 26 / 2023 \quad 02: 07: 18 \mathrm{PM}$ 


\section{Discussion}

In this study, we demonstrated that serum levels of FGF19, FGF21 and lipocalin-2 were significantly different between women with or without PCOS, and the joint effects of these biomarkers being associated with the risk of PCOS. We also showed that the effects of lipocalin-2 on diabetes risk might be different in PCOS and control subjects. Meanwhile, we also replicated the relationship between FGF19 and FGF21 with diabetes progression in normal individuals.

Our findings are in agreement with previous reports on the same tendency of increased FGF21 concentrations in PCOS women (22), even after adjusting for age and waist. FGF21 was positively associated with diabetes incidence or progression in the control group. However, both associations were attenuated after adjusting for baseline waist and FPG, which may be due to the limited sample size and strong association between FGF21 and the two variables. A meta-analysis of 28 studies reported elevated FGF21 concentration was associated with diabetes progression (hazard ratio (HR): 1.35, 95\% CI: 1.06-1.72, $P<0.05)$ (23). FGF21 has multiple beneficial effects on metabolism, insulin sensitivity and lipid homoeostasis in animal models. Increased FGF21 levels under metabolic stresses may result from increased circulating free-fatty acids and insulin (24). Moreover, adipose tissue inflammation in obesity, which involves the c-Jun NH2-terminal kinase 1 pathway, could result in the suppression of b-Klotho expression by TNF- $\alpha$ and hence impaired FGF21 action in adipocytes (25). FGF21 is also the upstream regulator of adiponectin. It can induce the expression and secretion of adiponectin in adipocytes to confer glucose-lowering and insulin-sensitizing effects (26). It is believed that the association between elevated FGF21 and risk of diabetes may reflect compensatory changes or a state of resistance to FGF21 $(27,28,29)$.

A similar relationship between FGF19 and diabetes incidence was also observed in the control group, though this was not found in the sensitivity analysisfor pre-diabetes. Several studies reported the tendency towards reduced FGF19 in people with diabetes $(30,31)$, but others reported there was no difference between T2DM and control (32). There are limited data from longitudinal studies on FGF19 and the prediction of diabetes. Serum FGF19 concentration was increased in our PCOS group, which was inconsistent with another study with decreased levels in PCOS women, but their sample size was comparatively small, and only 12 PCOS women were recruited (33).
Compared with controls, lipocalin-2 was decreased in PCOS subjects. Logistic regression analyses showed a strong negative association between serum lipocalin-2 levels and PCOS status. Previously, some studies reported similar effects with decreased lipocalin-2 in PCOS $(34,35)$, while others showed a tendency towards the opposite (36) or even no difference (37). Meanwhile, lipocalin-2 was associated with carotid intima-media thickness (35), waist, BMI (37) and insulin resistance (36); thus a difference in lipocalin-2 levels is plausible among different studies. Our study also identified novel associations between lipocalin-2 and diabetes progression in PCOS women. PCOS women had lower serum lipocalin-2 concentrations compared with those without PCOS, but lipocalin-2 was positively related to the incidence of T2DM in PCOS women, while negatively associated with diabetes in the control group. The different effects of lipocalin- 2 in the PCOS group vs the control group are intriguing, and may be due to different underlying mechanisms, including the effects of androgen and different body fat distribution.

Lipocalin-2 was associated with free testosterone; however, the trend of the association was inconsistent: Yilmaz et al. found that serum lipocalin-2 and free testosterone levels were positively correlated (38), while Diamanti-Kandarakis et al. reported a negative correlation (34). One study reported sexual dimorphism of lipocalin-2 expression in adipose tissue, and found that compared to control women, PCOS women (with androgen excess) had higher expression of lipocalin-2, with levels similar to those of men (39). Another animal study using mice with C57BL/6 background also found that lipocalin-2 had altered expression according to gender, as well as displaying age-related differences in expression in white adipose tissue (40). Moreover, in a large Chinese population-based cohort (median follow-up duration, 74 months), researchers could only find an association between baseline elevated lipocalin-2 and incident cardiovascular disease events among men, but not among women (41). All these studies are in line with the differential effects of lipocalin-2 according to levels of sex hormones, as observed in the current study. In the aforementioned animal study (40), the authors also found that lipocalin-2 had important effects on oestradiol biosynthesis and oestrogen receptor signalling in female mice, as mice with lipocalin-2 deficiency had significantly reduced expression levels of aromatase, which plays a key role in regulating oestradiol biosynthesis in adipose tissue, and these mice also showed lower levels of serum $17 \beta$-oestradiol and downregulated expression of oestrogen

This work is licensed under a Creative Commons Attribution-NonCommercial-NoDerivatives 4.0 Internationad bicense.ifica.com at 04/26/2023 02:07:18PM 
receptor $\alpha$ in adipose tissue after high-fat diet feeding or at old age (40). Nevertheless, despite these studies which support the differential effects of lipocalin-2 according to sex hormones, further studies are needed to investigate the specific mechanism of lipocalin-2 in PCOS women.

Importantly, subjects with elevated FGF19 and FGF21, together with decreased lipocalin-2, had 5.2-fold higher risk of PCOS than those with no more than one abnormal biomarker. After adjusting for common risk factors, the total number of abnormal biomarkers was still significantly associated with PCOS status in our cohort. Meanwhile, the increasing number of abnormal biomarkers were related to hypertension, hyperandrogenism and $\mathrm{AMH}$. Taken together, these findings support the possible utility of these biomarkers to identify subjects with PCOS. Our study has several strengths including the long-term follow-up and prospective data on incident diabetes in PCOS women and in normal Chinese women; the detailed phenotyping included evaluation of glycaemic status using an oral glucose tolerance test in each subject and the measurement of several novel biomarkers for metabolic risk. However, there are several limitations including the sample size being moderate with the limited number of incident diabetes during the follow-up period and the potential for over-fitting in our regression models. Our study is also limited by the drawback of a long-term follow-up study, whereby it is unavoidable that some participants were lost to follow-up due to various reasons. An immunoassay was used to measure insulin in the PCOS group while ELISA kits were used in the control group. The different measurements used in the two groups may have limited the opportunity to compare fasting insulin and HOMA-IR between the PCOS and control groups but should not affect the association between biomarkers and diabetes progression in those separate analyses. Besides, all data were from Chinese women lacking data from agematched men or women from other age groups, and hence replication in other studies with larger sample sizes and comparisons with other groups are needed.

In summary, our present results suggest altered circulating levels of FGF19, FGF21 and lipocalin-2 between PCOS women and healthy controls, and the combined effects of these biomarkers on the association with PCOS risk. We also highlighted the potential contribution of these biomarkers to established models for the prediction of diabetes progression in Chinese women with or without PCOS. Further studies in other populations may be needed to validate their use as possible biomarkers to identify high-risk subjects for interventions to prevent diabetes development.

\section{Supplementary materials}

This is linked to the online version of the paper at https://doi.org/10.1530/ EC-21-0082.

\section{Declaration of interest}

The authors declare that the research was conducted in the absence of any commercial or financial relationships that could be construed as a potential conflict of interest.

\section{Funding}

This study was partially supported by the Research Grants Council General Research Fund (ref. 14110415). N Y H N acknowledges support from the RGC Postdoctoral Fellowship Scheme.

\section{Author contribution statement}

$\mathrm{R} \mathrm{M}, J \mathrm{C}$, and L C contributed to the conception and design of the study. N N, $Y Z, C L$, and TY were responsible for clinical data acquisition and checking. $\mathrm{F} C$ and $\mathrm{A} N$ performed ELISA experiment. $\mathrm{A} X$ gave some guidance on experiment and data interpretation. F C, N N, C T, G J, and R M contributed to the data analysis, interpretation, and manuscript drafting. All authors read, revised, and approved the final manuscript.

\section{Acknowledgement}

The authors would like to express their sincere thanks to colleagues who assisted with patient recruitment and clinical assessment: Edith Kwok, Pearl Tsang.

\section{References}

1 Yau TT, Ng NY, Cheung LP \& Ma RC. Polycystic ovary syndrome: a common reproductive syndrome with long-term metabolic consequences. Hong Kong Medical Journal 201723 622-634. (https:// doi.org/10.12809/hkmj176308)

2 Li R, Zhang Q, Yang D, Li S, Lu S, Wu X, Wei Z, Song X, Wang X, Fu S, et al. Prevalence of polycystic ovary syndrome in women in China: a large community-based study. Human Reproduction 201328 2562-2569. (https://doi.org/10.1093/humrep/det262)

3 Cheung LP, Ma RCW, Lam PM, Lok IH, Haines CJ, So WY, Tong PCY, Cockram CS, Chow CC \& Goggins WB. Cardiovascular risks and metabolic syndrome in Hong Kong Chinese women with polycystic ovary syndrome. Human Reproduction 200823 1431-1438. (https://doi. org/10.1093/humrep/den090)

4 Moran LJ, Misso ML, Wild RA \& Norman RJ. Impaired glucose tolerance, type 2 diabetes and metabolic syndrome in polycystic ovary syndrome: a systematic review and meta-analysis. Human Reproduction Update 201016 347-363. (https://doi.org/10.1093/humupd/dmq001)

5 Gambineri A, Patton L, Altieri P, Pagotto U, Pizzi C, Manzoli L \& Pasquali R. Polycystic ovary syndrome is a risk factor for type 2 diabetes: results from a long-term prospective study. Diabetes 201261 2369-2374. (https://doi.org/10.2337/db11-1360)

6 Rubin KH, Glintborg D, Nybo M, Abrahamsen B \& Andersen M. Development and risk factors of type 2 diabetes in a nationwide population of women with polycystic ovary syndrome. Journal of Clinical Endocrinology and Metabolism 2017102 3848-3857. (https:// doi.org/10.1210/jc.2017-01354)

7 Ng NYH, Jiang G, Cheung LP, Zhang Y, Tam CHT, Luk AOY, Quan J, Lau ESH, Yau TTL, Chan MHM, et al. Progression of glucose intolerance 
and cardiometabolic risk factors over a decade in Chinese women with polycystic ovary syndrome: a case-control study. PLoS Medicine 201916 e1002953. (https://doi.org/10.1371/journal.pmed.1002953)

8 Jung U \& Choi M-S. Obesity and its metabolic complications: the role of adipokines and the relationship between obesity, inflammation, insulin resistance, dyslipidemia and nonalcoholic fatty liver disease. International Journal of Molecular Sciences 201415 6184-6223. (https:// doi.org/10.3390/ijms15046184)

9 Ouchi N, Parker JL, Lugus JJ \& Walsh K. Adipokines in inflammation and metabolic disease. Nature Reviews Immunology 201111 85-97. (https://doi.org/10.1038/nri2921)

10 Fu L, John LM, Adams SH, Yu XX, Tomlinson E, Renz M, Williams PM, Soriano R, Corpuz R, Moffat B, et al. Fibroblast growth factor 19 increases metabolic rate and reverses dietary and leptin-deficient diabetes. Endocrinology 2004145 2594-2603. (https://doi.org/10.1210/ en.2003-1671)

11 Izaguirre M, Gil MJ, Monreal I, Montecucco F, Fruhbeck G \& Catalan V. The role and potential therapeutic implications of the fibroblast growth factors in energy balance and Type 2 diabetes. Current Diabetes Reports 201717 43. (https://doi.org/10.1007/s11892-017-0866-3)

12 Liu JJ, Liu S, Choo RWM, Wee SL, Xu A \& Lim SC. Sex modulates the association of fibroblast growth factor 21 with end-stage renal disease in Asian people with type 2 diabetes: a 6.3-year prospective cohort study. Diabetic Medicine 201835 880-886. (https://doi.org/10.1111/ dme.13641)

13 Woo YC, Lee CH, Fong CHY, Xu A, Tso AWK, Cheung BMY \& Lam KSL. Serum fibroblast growth factor 21 is a superior biomarker to other adipokines in predicting incident diabetes. Clinical Endocrinology 2017 86 37-43. (https://doi.org/10.1111/cen.13229)

14 Lin Z, Wu Z, Yin X, Liu Y, Yan X, Lin S, Xiao J, Wang X, Feng W \& Li X. Serum levels of FGF-21 are increased in coronary heart disease patients and are independently associated with adverse lipid profile. PLOS ONE 20105 e15534. (https://doi.org/10.1371/journal.pone.0015534)

15 Lago F, Dieguez C, Gomez-Reino J \& Gualillo O. Adipokines as emerging mediators of immune response and inflammation. Nature Clinical Practice Rheumatology 20073 716-724. (https://doi. org/10.1038/ncprheum0674)

16 Revised 2003 consensus on diagnostic criteria and long-term health risks related to polycystic ovary syndrome. Fertility and Sterility 2004 81 19-25. (https://doi.org/10.1016/j.fertnstert.2003.10.004)

17 Ko GTC, Chan JCN, Chan AWY, Wong PTS, Hui SSC, Tong SDY, Ng S, Chow F \& Chan CLW. Association between sleeping hours, working hours and obesity in Hong Kong Chinese: the "better health for better Hong Kong' health promotion campaign. International Journal of Obesity 200731 254-260. (https://doi.org/10.1038/sj.ijo.0803389)

18 Levey AS, Stevens LA, Schmid CH, Zhang YL, Castro AF, 3rd, Feldman HI, Kusek JW, Eggers P, Van Lente F, Greene T, et al. A new equation to estimate glomerular filtration rate. Annals of Internal Medicine 2009150 604-612. (https://doi.org/10.7326/0003-4819-1509-200905050-00006)

19 Nathan DM, Buse JB, Davidson MB, Ferrannini E, Holman RR, Sherwin R \& Zinman B. Medical management of hyperglycemia in type 2 diabetes: a consensus algorithm for the initiation and adjustment of therapy: a consensus statement of the American Diabetes Association and the European Association for the Study of Diabetes. Diabetes Care 200932 193-203. (https://doi.org/10.2337/ dc08-9025)

20 McCartney CR \& Marshall JC. Polycystic ovary syndrome. New England Journal of Medicine 2016375 54-64. (https://doi.org/10.1056/ NEJMcp1514916)

21 Azziz R, Carmina E, Chen Z, Dunaif A, Laven JSE, Legro RS, Lizneva D, Natterson-Horowtiz B, Teede HJ \& Yildiz BO. Polycystic ovary syndrome. Nature Reviews Disease Primers 20162 1-18. (https://doi. org/10.1038/nrdp.2016.57)

22 Olszanecka-Glinianowicz M, Madej P, Wdowczyk M, Owczarek A \& Chudek J. Circulating FGF21 levels are related to nutritional status and metabolic but not hormonal disturbances in polycystic ovary syndrome. European Journal of Endocrinology 2015172 173-179. (https://doi.org/10.1530/EJE-14-0539)

23 Lakhani I, Gong M, Wong WT, Bazoukis G, Lampropoulos K, Wong SH, Wu WKK, Wong MCS, Ong K-L, Liu T, et al. Fibroblast growth factor 21 in cardio-metabolic disorders: a systematic review and meta-analysis. Metabolism 201883 11-17. (https://doi. org/10.1016/j.metabol.2018.01.017)

24 Woo YC, Xu A, Wang Y \& Lam KS. Fibroblast growth factor 21 as an emerging metabolic regulator: clinical perspectives. Clinical Endocrinology 201378 489-496. (https://doi.org/10.1111/cen.12095)

25 Diaz-Delfin J, Hondares E, Iglesias R, Giralt M, Caelles C \& Villarroya F. TNF-alpha represses beta-klotho expression and impairs FGF21 action in adipose cells: involvement of JNK1 in the FGF21 pathway. Endocrinology 2012153 4238-4245. (https://doi.org/10.1210/en.2012 1193)

26 Lin Z, Tian H, Lam KS, Lin S, Hoo RC, Konishi M, Itoh N, Wang Y, Bornstein SR, Xu A, et al. Adiponectin mediates the metabolic effects of FGF21 on glucose homeostasis and insulin sensitivity in mice. Cell Metabolism 201317 779-789. (https://doi.org/10.1016/j. cmet.2013.04.005)

27 Zhang X, Yeung DC, Karpisek M, Stejskal D, Zhou ZG, Liu F, Wong RL, Chow WS, Tso AW, Lam KS, et al. Serum FGF21 levels are increased in obesity and are independently associated with the metabolic syndrome in humans. Diabetes 200857 1246-1253. (https://doi. org/10.2337/db07-1476)

28 Reinehr T, Karges B, Meissner T, Wiegand S, Fritsch M, Holl RW \& Woelfle J. Fibroblast growth factor 21 and fetuin-A in obese adolescents with and without type 2 diabetes. Journal of Clinical Endocrinology and Metabolism 2015100 3004-3010. (https://doi.org/10.1210/jc.20152192)

29 Fisher FM, Chui PC, Antonellis PJ, Bina HA, Kharitonenkov A, Flier JS \& Maratos-Flier E. Obesity is a fibroblast growth factor 21 (FGF21)resistant state. Diabetes 201059 2781-2789. (https://doi.org/10.2337/ db10-0193)

30 Barutcuoglu B, Basol G, Cakir Y, Cetinkalp S, Parildar Z, Kabaroglu C, Ozmen D, Mutaf I \& Bayindir O. Fibroblast growth factor-19 levels in type 2 diabetic patients with metabolic syndrome. Annals of Clinical and Laboratory Science 201141 390-396.

31 Fang Q, Li H, Song Q, Yang W, Hou X, Ma X, Lu J, Xu A \& Jia W. Serum fibroblast growth factor 19 levels are decreased in Chinese subjects with impaired fasting glucose and inversely associated with fasting plasma glucose levels. Diabetes Care 201336 2810-2814. (https://doi. org/10.2337/dc12-1766)

32 Mráz M, Lacinová Z, Kaválková P, Haluzikova D, Trachta P, Drapalova J, Haluzík M \& Hanušová V. Serum concentrations of fibroblast growth factor 19 in patients with obesity and type 2 diabetes mellitus: the influence of acute hyperinsulinemia, very-low calorie diet and PPAR- $\alpha$ agonist treatment. Physiological Research 201160 627-636. (https://doi. org/10.33549/physiolres.932099)

33 Wang D, Zhu W, Li J, An C \& Wang Z. Serum concentrations of fibroblast growth factors 19 and 21 in women with gestational diabetes mellitus: association with insulin resistance, adiponectin, and polycystic ovary syndrome history. PLoS ONE 20138 e81190. (https:// doi.org/10.1371/journal.pone.0081190)

34 Diamanti-Kandarakis E, Livadas S, Kandarakis SA, Margeli A \& Papassotiriou I. Serum concentrations of atherogenic proteins neutrophil gelatinase-associated lipocalin and its complex with matrix metalloproteinase- 9 are significantly lower in women with polycystic ovary syndrome: hint of a protective mechanism? European Journal of Endocrinology 2008158 525-531. (https://doi.org/10.1530/EJE-070822)

35 Gencer M, Gazi E, Hacıvelioğlu S, Binnetoğlu E, Barutçu A, Türkön H, Temiz A, Altun B, Vural A, Cevizci S, et al. The relationship between subclinical cardiovascular disease and lipocalin-2 levels in women with PCOS. European Journal of Obstetrics, Gynecology, and 
Reproductive Biology 2014181 99-103. (https://doi.org/10.1016/j. ejogrb.2014.07.032)

36 Cakal E, Ozkaya M, Engin-Ustun Y \& Ustun Y. Serum lipocalin-2 as an insulin resistance marker in patients with polycystic ovary syndrome. Journal of Endocrinological Investigation 201134 97-100. (https://doi. org/10.1007/BF03347037)

37 Panidis D, Tziomalos K, Koiou E, Kandaraki EA, Tsourdi E, Delkos D, Kalaitzakis E \& Katsikis I. The effects of obesity and polycystic ovary syndrome on serum lipocalin-2 levels: a cross-sectional study. Reproductive Biology and Endocrinology 20108 151. (https://doi. org/10.1186/1477-7827-8-151)

38 Yilmaz Ö, Temur M, Calan M, Kume T, Özbay PÖ, Karakulak M \& Yapucu S. The relationship between lipocalin-2 and free testosterone levels in polycystic ovary syndrome. Endokrynologia Polska 201768 7-12. (https://doi.org/10.5603/EP.2017.0002)
39 Martínez-García MÁ, Montes-Nieto R, Fernández-Durán E, Insenser M, Luque-Ramírez M \& Escobar-Morreale HF. Evidence for masculinization of adipokine gene expression in visceral and subcutaneous adipose tissue of obese women with polycystic ovary syndrome (PCOS). Journal of Clinical Endocrinology and Metabolism 201398 E388-E396. (https://doi.org/10.1210/jc.2012-3414)

40 Guo H, Zhang Y, Brockman DA, Hahn W, Bernlohr DA \& Chen X. Lipocalin 2 deficiency alters estradiol production and estrogen receptor signaling in female mice. Endocrinology 2012153 1183-1193. (https://doi.org/10.1210/en.2011-1642)

41 Wu G, Li H, Fang Q, Jiang S, Zhang L, Zhang J, Hou X, Lu J, Bao Y, Xu A, et al. Elevated circulating lipocalin-2 levels independently predict incident cardiovascular events in men in a population-based cohort. Arteriosclerosis, Thrombosis, and Vascular Biology 201434 2457-2464. (https://doi.org/10.1161/ATVBAHA.114.303718)

Received in final form 25 February 2021

Accepted 2 September 2021

Accepted Manuscript published online 2 September 2021
This work is licensed under a Creative Commons Attribution-NonCommercial-NoDerivatives 4.0 Internationablicense $i f i c a . c o m$ at $04 / 26 / 2023$ 02:07:18PM 